of Rheumatology, Great Neck, United States of America; ${ }^{5}$ Monash University, Centre for Inflammatory Disease Monash Health, Melbourne, Australia; ${ }^{6}$ University of Occupational and Environmental Health, Japan, First Department of Internal Medicine, Kitakyushu, Japan; ${ }^{7}$ Lupus Center of Excellence, Autoimmunity Institute, Allegheny Health Network, Rheumatology, Pittsburgh, United States of America; ${ }^{8}$ University of California, San Diego, Division of Rheumatology, Allergy and Immunology, La Jolla, United States of America; ${ }^{9}$ AstraZeneca, BioPharmaceuticals R\&D, Gaithersburg, United States of America; ${ }^{10}$ AstraZeneca, BioPharmaceuticals R\&D, Cambridge, United Kingdom

Background: Patients with systemic lupus erythematosus (SLE) who received anifrolumab, a type I interferon receptor antibody, had greater BILAG-based Composite Lupus Assessment (BICLA) response rates vs placebo at Week (W)52 in the phase 2 MUSE $^{1}$ and the phase 3 TULIP- $1^{2}$ and TULIP- $2^{3}$ trials. Patients receiving anifrolumab also had fewer flares, and more patients were able to taper glucocorticoids (GC) vs placebo. ${ }^{1-3}$

Objectives: To evaluate anifrolumab treatment response vs placebo in patients with SLE from MUSE, TULIP-1, and TULIP-2 using more stringent BICLA definitions, as well as a novel endpoint that requires dual BICLA and SLE Responder Index (SRI[4]) responses.

Methods: MUSE (NCT01438489), TULIP-1 (NCT02446912), and TULIP-2 (NCT02446899) were randomized, placebo-controlled, 52-week trials of intravenous anifrolumab (every 4 weeks for 48 weeks) in patients with moderate to severe SLE despite standard therapy. ${ }^{1-3}$ Sustained GC taper was defined as taper to $\leq 7.5 \mathrm{mg} /$ day in patients receiving $\mathrm{GC} \geq 10 \mathrm{mg} /$ day at baseline, or to less than or equal to baseline dose in patients receiving $\mathrm{GC}<10 \mathrm{mg} /$ day at baseline, achieved by $\mathrm{W} 40$ and sustained through W52. Response rates were compared between anifrolumab $300 \mathrm{mg}$ vs placebo groups for patients who 1) attained a W52 BICLA response with sustained GC taper; 2) attained a W52 BICLA response and no flares after W12 (flare defined as $\geq 1$ new BILAG-2004 A or $\geq 2$ new BILAG-2004 $B$ scores vs the prior visit); 3) attained a W52 BICLA response with sustained GC taper and no flares after W12; 4) attained an enhanced BICLA (eBICLA) response at W52 that required complete resolution of all baseline BILAG-2004 activity (all baseline A/B scores to D; no worsening of $C$ or D scores); and 5) met both BICLA and SRI(4) response criteria.

Results: Evaluated patients received anifrolumab $300 \mathrm{mg}$ (MUSE, $n=99$; TULIP-1 and TULIP-2, $\mathrm{n}=180$ ) or placebo (MUSE, $\mathrm{n}=102$; TULIP-1, $\mathrm{n}=184$; TULIP-2, $n=182$ ). Response rate differences favoring anifrolumab $300 \mathrm{mg}$ over placebo were observed for all 5 endpoints across MUSE, TULIP-1, and TULIP-2 (Figure 1). A greater proportion of patients had BICLA responses at W52 with sustained GC taper with anifrolumab vs placebo. More patients had BICLA responses at W52 with no flares after W12 with anifrolumab vs placebo. More patients had BICLA responses at W52 with both sustained GC taper and no flares after W12 with anifrolumab vs placebo (treatment difference, 15.3\%19.3\%; nominal $P \leq 0.006$ ). More patients attained eBICLA responses (requiring complete resolution of baseline disease activity) at W52 with anifrolumab vs placebo (treatment difference, $11.1 \%-14.1 \%$; nominal $P \leq 0.017$ ). In addition, more

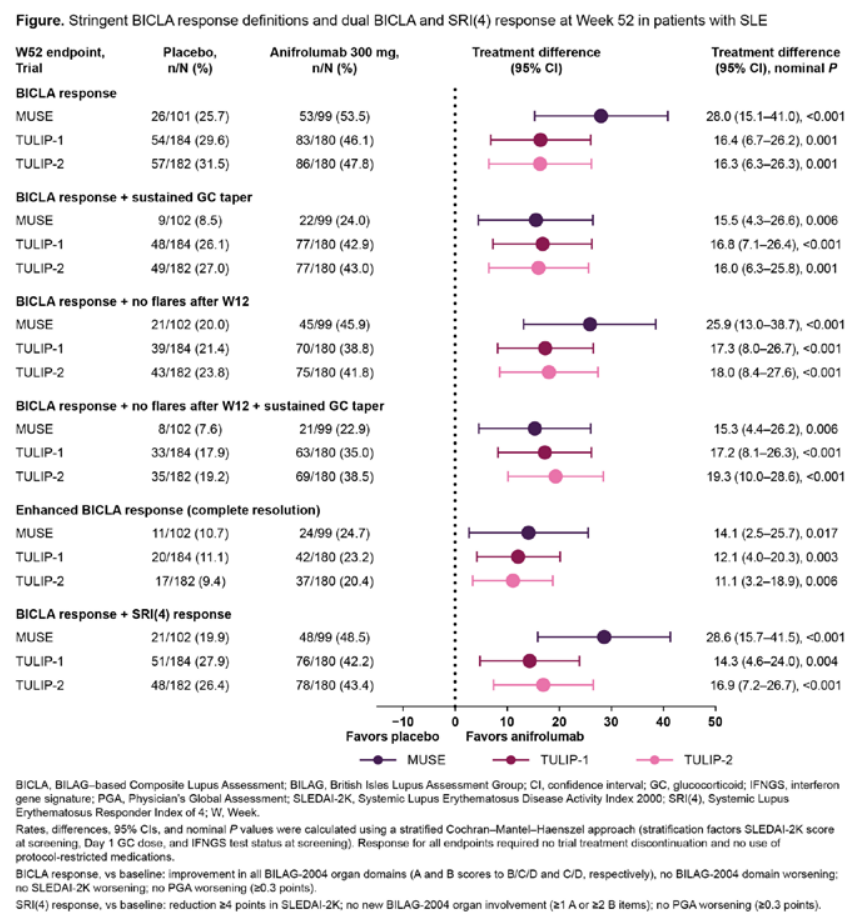

patients were dual BICLA and SRI(4) responders at W52 with anifrolumab vs placebo (treatment difference, $14.3 \%-28.6 \%$; nominal $P \leq 0.004$ ).

Conclusion: In phase 2 and 3 trials in patients with SLE, anifrolumab treatment was consistently associated with improved disease control vs placebo using stringent endpoint definitions, including BICLA response with sustained GC taper and no flares, an enhanced BICLA response requiring complete resolution of baseline disease activity, and dual BICLA and SRI(4) responses. REFERENCES:

[1] Furie R, et al. Arthritis Rheumatol. 2017;69:376-86.

[2] Furie RA, et al. Lancet Rheumatol. 2019;1:e208-19.

[3] Morand EF, et al. N Engl J Med. 2020;382:211-21.

Acknowledgements: Writing assistance by Rosie Butler, PhD, of JK Associates Inc., part of Fishawack Health. This study was sponsored by AstraZeneca.

Disclosure of Interests: David Isenberg Consultant of: AstraZeneca, Celgene, Merck Serono, UCB, and Servier, Ian N. Bruce Speakers bureau: AstraZeneca, GSK, and UCB, Consultant of: Eli Lilly, GSK, ILTOO, Merck Serono, and UCB, Grant/research support from: Genzyme/Sanofi, GSK, Roche, and UCB, Richard Furie Consultant of: AstraZeneca, Grant/research support from: AstraZeneca, Eric F. Morand Speakers bureau: AstraZeneca, Consultant of: AstraZeneca, Grant/ research support from: AstraZeneca, Yoshiya Tanaka Speakers bureau: AbbVie, Asahi Kasei, Astellas, Bristol Myers Squibb, Chugai, Daiichi-Sankyo, Eisai, Eli Lilly, Gilead, GSK, Janssen, Mitsubishi-Tanabe, Novartis, Pfizer, Sanofi, and YL Biologics, Grant/research support from: AbbVie, Chugai, Daiichi-Sankyo, Eisai, Mitsubishi-Tanabe, Takeda, and UCB, Susan Manzi Consultant of: AstraZeneca, Grant/research support from: AstraZeneca, Kenneth Kalunian Consultant of: AstraZeneca, Konstantina Psachoulia Employee of: AstraZeneca, Emmanuelle Maho Employee of: AstraZeneca, Raj Tummala Employee of: AstraZeneca

DOI: 10.1136/annrheumdis-2021-eular.702

\section{POS0684 RELATIONSHIP OF ANIFROLUMAB PK WITH EFFICACY AND SAFETY IN PATIENTS WITH SLE}

Y. L. Chia ${ }^{1}$, J. Zhang ${ }^{2}$, R. Tummala ${ }^{3}$, T. Rouse ${ }^{4}$, R. Furie ${ }^{5}$, E. F. Morand ${ }^{6}$. ${ }^{1}$ AstraZeneca, BioPharmaceuticals $R \& D$, South San Francisco, United States of America; ${ }^{2}$ Fate Therapeutics Inc, Department of Data Science, San Diego, United States of America; ${ }^{3}$ AstraZeneca, BioPharmaceuticals R\&D, Gaithersburg, United States of America; ${ }^{4}$ AstraZeneca, BioPharmaceuticals $R \& D$, Gothenburg, Sweden; ${ }^{5}$ Zucker School of Medicine at Hofstra/Northwell, Division of Rheumatology, Great Neck, United States of America; ${ }^{6}$ Monash University, Centre for Inflammatory Disease Monash Health, Melbourne, Australia

Background: In patients with systemic lupus erythematosus (SLE), the type I interferon (IFN) receptor inhibitor anifrolumab was well tolerated and was associated with greater percentages of patients with BILAG-based Composite Lupus Assessment (BICLA) responses vs placebo in 2 phase 3 trials: TULIP- ${ }^{1}$ (secondary endpoint) and TULIP-2 ${ }^{2}$ (primary endpoint).

Objectives: To characterize the relationship of anifrolumab pharmacokinetics (PK) with BICLA response and safety using pooled data from the TULIP trials Methods: This analysis included patients with moderate to severe SLE despite standard therapy who had $\geq 1$ dose of investigational product and $\geq 1$ quantifiable PK observation in the randomized, placebo-controlled, 52-week TULIP-1 (NCT02446912) and TULIP-2 (NCT02446899) trials of intravenous anifrolumab (every 4 weeks). ${ }^{1,2}$ The distributions of average anifrolumab serum concentrations (C $)$ during treatment were similar between TULIP-1 and TULIP-2, allowing for data pooling for all analyses. For the exposure-BICLA analysis, the proportions of patients with BICLA responses at Week (W)52 (and corresponding 95\% confidence intervals [Cls]) in each quartile/tertile of $\mathrm{C}_{\text {ave }}$ were compared for anifrolumab $300 \mathrm{mg}$ and placebo groups in all patients, patients who completed treatment, and IFN gene signature (IFNGS) test-high patients who completed treatment, using average marginal effect logistic regression (stratified by SLE Disease Activity Index 2000 total score at screening, IFNGS status at screening, and Day 1 glucocorticoid dosage [mg/day]). The relationships between exposure and key safety events were similarly assessed. Analyses presented focus on the anifrolumab $300 \mathrm{mg}$ dose. Results: Of the patients in TULIP-1/TULIP-2 who received anifrolumab $300 \mathrm{mg}$ $(n=356)$ or placebo $(n=366), 574$ completed treatment, of whom 470 were IFNGS test-high at screening. In the exposure-BICLA response analyses, differences favoring anifrolumab $300 \mathrm{mg}$ vs placebo were observed across $C$ subgroups among all patients, patients who completed treatment, and IFNGS test-high patients who completed treatment (Table 1). Among IFNGS test-high patients who completed treatment, logistic regression identified $\mathrm{C}_{\text {ave }}$ as a significant covariate for BICLA response. There was no evidence that the incidence of non-opportunistic serious infections, or increased incidence of herpes zoster $(\mathrm{HZ})$ or infusion-related reactions associated with anifrolumab, were exposure-driven (Figure 1); the incidence of malignancy was low in the anifrolumab $300 \mathrm{mg}$ and placebo groups $(<1 \%)$, with no evidence that malignancy was exposure-driven through W52. 
Conclusion: Consistent benefit in favor of anifrolumab $300 \mathrm{mg}$ vs placebo was observed in W52 BICLA responses across $C_{\text {ave }}$ subgroups. $C_{\text {ave }}$ was a significant covariate of efficacy in IFNGS test-high patients who completed treatment. There was no evidence of exposure-driven $\mathrm{HZ}$, non-opportunistic serious infections, infusion-related reactions, or malignancy during the TULIP trials.

REFERENCES:

[1] Furie R. Lancet Rheumatol. 2019;1:e208-19.

[2] Morand E. N Engl J Med. 2020;382:211-21.

Table 1. Exposure-BICLA Analysis for Pooled TULIP Data

\begin{tabular}{|c|c|c|c|}
\hline BICLA response, W52 & $\begin{array}{l}\text { PK } \\
\text { subgroup }\end{array}$ & $\begin{array}{l}\text { Anifrolumab } \\
300 \mathrm{mg} \text {, } \\
\mathrm{n} / \mathrm{N}^{\mathrm{b}}(\%)\end{array}$ & $\begin{array}{l}\text { Anifrolumab vs placebo } \\
\text { difference, } \%[95 \% \mathrm{Cl}]\end{array}$ \\
\hline All patients $(n=722)$ & $\begin{array}{l}\text { Q1 } \\
\text { Q2 } \\
\text { Q3 } \\
\text { Q4 } \\
\text { Placebo }\end{array}$ & $\begin{array}{l}40 / 100(40) \\
44 / 98(44) \\
43 / 81(53) \\
44 / 77(58) \\
112 / 366(31)\end{array}$ & $\begin{array}{l}9.6[-1.0,20.3] \\
13.4[2.6,24.2] \\
22.5[10.7,34.3] \\
27.4[15.4,39.4] \\
-\end{array}$ \\
\hline $\begin{array}{l}\text { Patients completing treatment } \\
\quad(n=574)\end{array}$ & $\begin{array}{l}\text { Q1 } \\
\text { Q2 } \\
\text { Q3 } \\
\text { Q4 } \\
\text { Placebo }\end{array}$ & $\begin{array}{l}40 / 75(54) \\
44 / 74(57) \\
43 / 74(58) \\
44 / 75(60) \\
112 / 276(41)\end{array}$ & $\begin{array}{l}12.7[0.1,25.2] \\
15.5[2.7,28.3] \\
17.2[4.7,29.8] \\
18.7[6.2,31.2] \\
-\end{array}$ \\
\hline $\begin{array}{l}\text { IFNGS test-high patients com- } \\
\text { pleting treatment }(n=470)\end{array}$ & $\begin{array}{l}\text { T1 } \\
\text { T2 } \\
\text { T3 } \\
\text { Placebo }\end{array}$ & $\begin{array}{l}44 / 81(54) \\
46 / 81(54) \\
52 / 81(66) \\
88 / 227(39)\end{array}$ & $\begin{array}{l}15.4[3,27.8] \\
15.4[2.8,27.9] \\
26.7[14.7,38.7] \\
-\end{array}$ \\
\hline
\end{tabular}

BICLA, British Isles Lupus Assessment Group-based Composite Lupus Assessment; $\mathrm{Cl}$ confidence interval; IFNGS, interferon gene signature; PK, pharmacokinetic; $Q$, quartile; $\mathrm{T}$, tertile. ${ }^{a} \mathrm{PK}$ was stratified by quartiles/tertiles based on sample size. ${ }^{\mathrm{b}} \mathrm{n}$, number of BICLA responders; $\mathrm{N}$, number of patients in the subgroup.

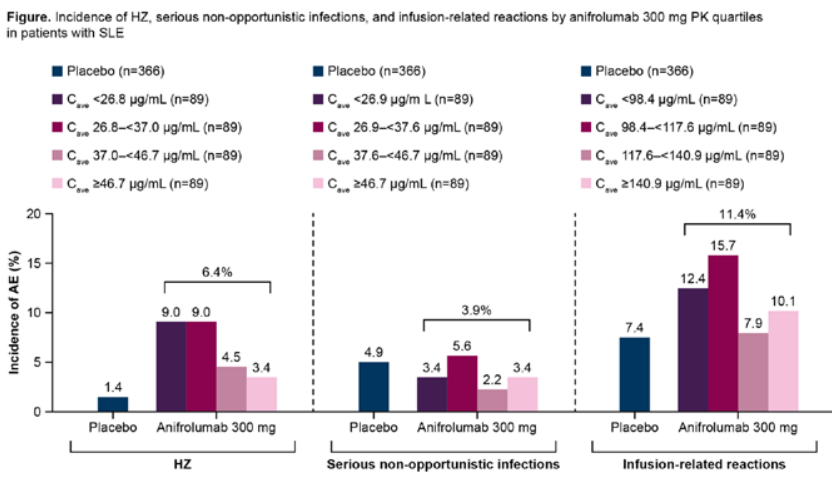

Acknowledgements: Writing assistance by Alexus Rivas, PharmD, and Rosie Butler, PhD, of JK Associates Inc., part of Fishawack Health.

This study was sponsored by AstraZeneca.

Disclosure of Interests: Yen Lin Chia Employee of: AstraZeneca, Jianchun Zhang Employee of: Fate Therapeutics, AstraZeneca (former), Raj Tummala Employee of: AstraZeneca, Tomas Rouse Employee of: AstraZeneca, Richard Furie Consultant of: AstraZeneca, Grant/research support from: AstraZeneca, Eric F. Morand Speakers bureau: AstraZeneca, Consultant of: AstraZeneca, Grant/research support from: AstraZeneca

DOI: 10.1136/annrheumdis-2021-eular.803

\begin{tabular}{|l|l}
\hline POS0685 & MYCOPHENOLATE MOFETIL IN SYSTEMIC LUPUS \\
& ERYTHEMATOSUS PATIENTS: FIVE-YEARS DRUG \\
& SURVIVAL IN RENAL AND NON-RENAL INVOLVEMENT
\end{tabular}

G. Olivieri ${ }^{1}$, F. Ceccarelli ${ }^{1}$, F. Natalucci ${ }^{1}$, F. R. Spinelli ${ }^{1}$, C. Alessandri ${ }^{1}$, F. Conti ${ }^{1}$. ${ }^{1}$ Lupus Clinic, Sapienza Università di Roma, Dipartimento di Scienze Cliniche Internistiche, Anestesiologiche e Cardiovascolari, Roma, Italy

Background: The updated EULAR recommendations for the management of systemic lupus erythematosus (SLE) underline the use of Mycophenolate Mofetil (MMF) in the treatment of different disease related manifestations (1). Several randomized controlled trials have demonstrated the efficacy of MMF in lupus nephritis (LN) patients but only case series and open-labelled trials have analyzed the use of this drug in other than LN features. Moreover, no data are available about the MMF retention rate in a real-life setting.
Objectives: The present study aims at evaluating the 5-years drug retention rate (DRR) of MMF in a large monocentric SLE cohort. Secondly, we investigated the influence of MMF in disease activity changes and chronic damage progression. Methods: We performed a longitudinal study including all the SLE patients (ACR 1997 criteria) starting MMF treatment in our Lupus Clinic. Data about indications, mean dosage, duration of treatment and reasons for drug withdrawal were registered. The DRR was estimated using the Kaplan-Meier method. Disease activity and chronic damage were assessed by SLE Disease Activity Index 2000 (SLEDAI-2K) and SLICC Damage Index (SDI), respectively.

Results: The present analysis included 162 SLE patients (M/F 22/140, median age at the disease diagnosis 25.5 years, IQR 13). At the beginning of MMF treatment, we registered a median age of 34 months (IQR 21) and a median disease duration of 72 months (IQR 123). The most frequent indications for prescribing MMF were LN (101 patients, $62.3 \%$ ) and musculoskeletal manifestations (39, 24.1\%), followed by neuropsychiatric involvement $(10,6.2 \%)$, and others disease related manifestations (12,7.4\%; in particular skin involvement, hematological features, myositis, vasculitis). MMF was administered at a mean daily dosage of $2.1 \pm 0.6$ grams; no differences in dosage were found between the different indications $(p=n s)$.

At the longitudinal analysis, we registered a median treatment duration of 30 months (IQR 55). Figure 1 reported data about DRR: in particular, at 60 months follow-up we observed a DRR of $61.1 \%$ for LN patients, which was similar to that registered for patients without renal involvement (NLN) $(60.5 \%$; $p=n s)$. Interestingly, the DRR at 60 months was higher in the subgroup of patients treated for joint involvement $(75.4 \%)$, even without reaching a statistically significant difference. During the observation period, 92 patients $(59.2 \%)$ discontinued MMF (median treatment duration at discontinuation 25 months, IQR 35). Interestingly, the main cause of withdrawal was the achievement of persistent remission, observed in 20 patients (21.7\%), followed by loss of efficacy (19 patients, $20.5 \%$ ), drug intolerance and pregnancy planning (17 patients for both reasons, 18,4\%). Furthermore, our analysis confirmed MMF efficacy, as demonstrated by the significant reduction in SLEDAI-2k values after 4, 12 and 24 months of treatment $(p<0.0001$ for all the time-points in comparison with baseline). In addition, MMF resulted able to control chronic damage progression, as demonstrated by the lack of significant increase in SDI values (baseline: 0.6 , IQR 1; last observation: 0.93, IQR 1; $\mathrm{p}=\mathrm{ns}$ )

Conclusion: The evaluation of a large SLE cohort demonstrated a good retention rate for MMF. In particular, our results demonstrated that MMF is also a safe and effective drug for SLE manifestation other than LN, in particular for joint involvement. Moreover, it is able to control disease activity and to prevent the progression of chronic damage.

REFERENCES:

[1] Fanouriakis A et al. Ann Rheum Dis. 2019 Jun;78(6):736-745.
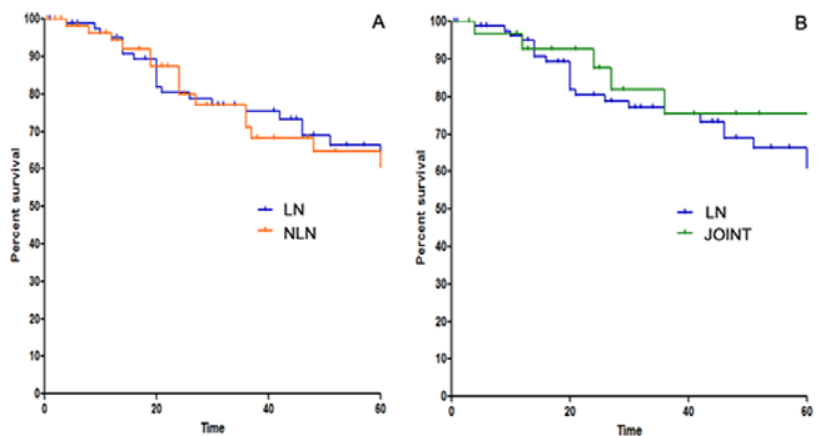

Figure 1. Kaplan-Meier survival curve of MMF treatment. The time of MMF treatment is expressed as months. DRR of MMF therapy at 60 months was similar in LN patients and NLN patients (Figure $1 \mathrm{~A}$ ). Interestingly, the difference (Figure 1B).

Disclosure of Interests: None declared

DOI: 10.1136/annrheumdis-2021-eular.925

\section{POS0686}

\section{BARICITINIB DECREASES ANTI-DSDNA AND IGG ANTIBODIES IN ADULTS WITH SYSTEMIC LUPUS ERYTHEMATOSUS FROM A PHASE 2 DOUBLE-BLIND, RANDOMIZED, PLACEBO-CONTROLLEDTRIAL}

T. Dörner ${ }^{1}$, R. Van Vollenhoven ${ }^{2}$, A. Doria ${ }^{3}$, B. Jia ${ }^{4}$, D. Fantini ${ }^{4}$, J. Ross Terres ${ }^{4}$, M. Silk ${ }^{4}$, S. De Bono ${ }^{4}$, P. Fischer ${ }^{4}$, D. J. Wallace ${ }^{5} .{ }^{1}$ Charite Universitätsmedizin Berlin and Deutsches Rheumaforschungszentrum (DRFZ), Division of Rheumatology, Berlin, Germany; ${ }^{2}$ University Medical Center, Department of Rheumatology, Amsterdam, Netherlands; ${ }^{3}$ University of Padova, Department of Medicine, Padova, Italy; ${ }^{4}$ Eli Lilly and Company, Lilly Research Laboratories, Indianapolis, United States of America; ${ }^{5}$ Cedars-Sinai Medical Centerl University California at Los Angeles, Division of Rheumatology, Los Angeles, United States of America 